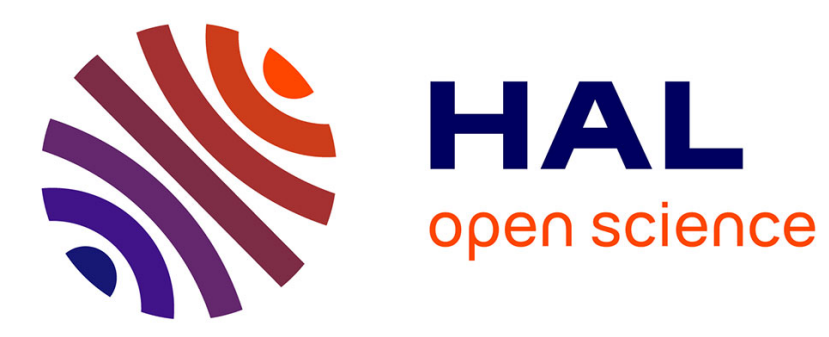

\title{
Numerical simulation of spreading drops
}

Dominique Legendre, Marco Maglio

\section{To cite this version:}

Dominique Legendre, Marco Maglio. Numerical simulation of spreading drops. Colloids and Surfaces A: Physicochemical and Engineering Aspects, 2013, vol. 432, pp.29-37. 10.1016/j.colsurfa.2013.04.046 . hal-00919690

\section{HAL Id: hal-00919690 https://hal.science/hal-00919690}

Submitted on 17 Dec 2013

HAL is a multi-disciplinary open access archive for the deposit and dissemination of scientific research documents, whether they are published or not. The documents may come from teaching and research institutions in France or abroad, or from public or private research centers.
L'archive ouverte pluridisciplinaire HAL, est destinée au dépôt et à la diffusion de documents scientifiques de niveau recherche, publiés ou non, émanant des établissements d'enseignement et de recherche français ou étrangers, des laboratoires publics ou privés. 


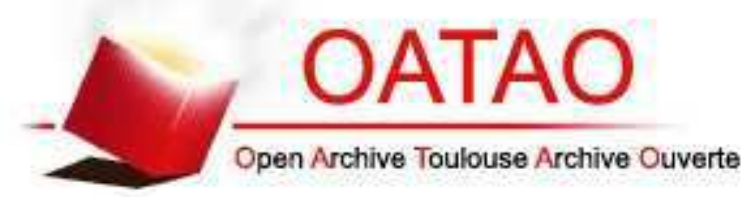

\section{Open Archive TOULOUSE Archive Ouverte (OATAO)}

OATAO is an open access repository that collects the work of Toulouse researchers and makes it freely available over the web where possible.

This is an author-deposited version published in : http://oatao.univ-toulouse.fr/ Eprints ID : 10539

\section{To link to this article :}

DOI:10.1016/j.colsurfa.2013.04.046

URL : http://dx.doi.org/10.1016/j.colsurfa.2013.04.046

\section{To cite this version :}

Legendre, Dominique and Maglio, Marco Numerical simulation of spreading drops. (2013) Colloids and Surfaces A: Physicochemical and Engineering Aspects, vol. 432 . pp. 29-37. ISSN 0927-7757

Any correspondance concerning this service should be sent to the repository administrator: staff-oatao@listes-diff.inp-toulouse.fr 


\title{
Numerical simulation of spreading drops
}

\author{
Dominique Legendre*, Marco Maglio* \\ Institut de Mécanique des Fluides de Toulouse (IMFT), Université de Toulouse, CNRS-INPT-UPS, France
}

\section{G R A P H I C A L A B S T R A C T}

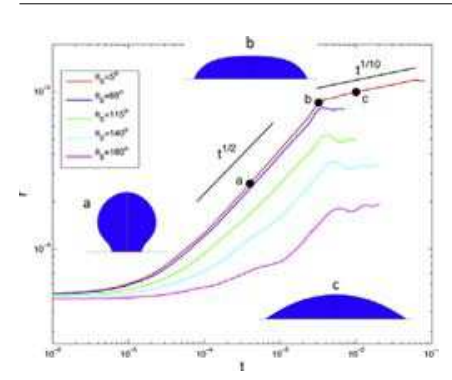

- Numerial simulation of spreading drops on a wettable surface.

- We discuss the effect of the main parameters (density, viscosity, surface tension, drop radius and wettability).

- Power law evolutions observed in experiments $\left(t^{1} / 2, t^{1} / 10\right)$ are recovered and discussed.

- The combined effect of viscosity and wettability has a significant effect on the spreading.

Keywords:

Drop spreading

Numerical simulation

\begin{abstract}
A B S T R A C T
We consider a liquid drop that spreads on a wettable surface. Different time evolutions have been observed for the base radius $r$ depending of the relative role played by inertia, viscosity, surface tension and the wetting condition. Numerical simulations were performed to discuss the relative effect of these parameters on the spreading described by the evolution of the base radius $r(t)$ and the spreading time $t_{S}$. Different power law evolutions $r(t) \propto t^{n}$ have been observed when varying the parameters. At the early stage of the spreading, the power law $t^{1 / 2}(n=1 / 2)$ is observed as long as capillarity is balanced by inertia at the contact line. When increasing the viscosity contribution, the exponent $n$ is found to increase despite the increase of the spreading time. The effect of the surface wettability is observed for liquids more viscous than water. For a small contact angle, the power law $t^{1 / 2}$ is then followed by the famous Tanner law $t^{1 / 10}$ once the drop shape has reached a spherical cap.
\end{abstract}

\section{Introduction}

When a liquid drop contacts a wettable surface, the liquid spreads over the solid to minimize the total surface energy. The first moments of spreading tend to be rapid (some milli-seconds for millimeter sized water drops) while it takes a much longer time for viscous liquids to completely spread. From the literature $[22,17,9,1,6,3,2,24,7]$, it appears that several time evolutions can be observed for the base radius depending if inertia, viscosity and/or

\footnotetext{
* Corresponding authors. Tel.: +33 5343228 18; fax: +33 534322991 .

E-mail address: legendre@imft.fr (D. Legendre).
}

gravity are involved. In addition, the effect of the substrate wettability on drop spreading is not yet clearly understood.

The evolution of the wetted surface is reported using either the base radius $r(t)$ or the wetted surface $A(t)=\pi r^{2}(t)$. The experimental results are usually presented in order to provide a power law evolution under the form:

$r(t) \sim t^{n}$, or $A(t) \sim t^{2 n}$,

The most popular law for drop spreading is certainly the so-called Tanner law $[23,22]$ with $n=1 / 10$. This evolution can be simply obtained by considering that the shape of the drop is a thin spherical cap and that the contact angle follows the Cox-Voinov relation. Such evolution has been found to be in agreement with several 
experiments (see [3]). The Tanner law concerns spreading situations in which surface tension is balanced by viscosity. Varying the relative different contributions (inertia, gravity, viscosity, surface tension) different evolutions have been reported: $n=0.1, n=0.14$, $n=0.16, n=1 / 8, n=1 / 7, n=1 / 2[17,9,3]$. The $t^{1 / 2}$ power law results from a balance between the capillary pressure and the inertial pressure. Such evolution has been observed in several experiments $[1,2,24]$. Lavi and Marmur [15] performed experiments for different liquids on a coated silicon wafer and reported the following exponential evolution:

$\frac{A(t)}{A_{f}}=1-\exp \left[-\frac{K}{A_{f}} \tau^{m}\right]$,

where $A_{f}$ is the final value of the wetted area and $\tau$ is the dimensionless time $\tau=\sigma t / \mu V^{1 / 3}$. Lavi and Marmur [15] reports values for $m$ in the range $m=0.618-0.964$. The interest of this relation is to propose a power law of the form $t^{m}$ in the limit of large final wetted surface $A_{f}$. As it will be shown in the simulations reported in this paper, the viscosity has an important effect on the drop spreading. For low viscous liquids (as water), a power law of the form (1) can be observed during almost all the evolution. When increasing the viscosity, the slope of the evolution changes over time, showing an exponential dependency of the form (2). The wettability has also an impact on the drop spreading. The distinction between the different wetting states is usually made by considering the equilibrium spreading coefficient $S_{\text {eq }}=\sigma\left(\cos \theta_{S}-1\right)$, which represents the surface free energy relative to its value for complete wetting. When a drop is deposited on a plane surface, the initial contact angle $\theta_{i} \approx 180^{\circ}$ made by the interface with the wall is larger than equilibrium $\theta_{S}$. An initial spreading coefficient $S_{i}=\sigma\left(\cos \theta_{i}-1\right)$ can be introduced to characterize the initial energy of the system. The difference $\Delta S=S_{\text {eq }}-S_{i}=\cos \theta_{S}-\cos \theta_{i}$ indicates the nature of the spreading. Since $\Delta S<0$, the drop spreads until the contact angle stabilizes at the equilibrium angle $\theta_{S}$. Neither different theoretical models nor experimental observations have shown a direct effect of $\Delta S=\cos \theta_{S}-\cos \theta_{i}$ on the drop spreading. Furthermore, the impact of the surface wettability on the drop spreading is still under controversy. Biance et al. [1] observed the scaling law $r(t) \sim t^{1 / 2}$ in their experiments of water drops spreading on a completely wetting surface. Bird et al. [2] performed experiments with droplets of different water-glycerol mixtures $(1.0,3.7$, and $10.7 \mathrm{cP})$ on silicon wafer surfaces of variable wettability (from $\theta_{S}=0^{\circ}$ to $\theta_{S}=180^{\circ}$ ). The spreading radius is found to follow a power-law evolution of the form (1) where the exponent $n$ depends on the equilibrium contact angle. Typically it varies from $n=1 / 2$ for perfect wetting surfaces to $n=1 / 4$ for non wetting surfaces. Chen et al. [7] observed spreading of water drop on hydrophobic glass with $n=0.3$. On the contrary, the recent experiments of Winkels et al. [24] point toward a spreading regime that is independent of wettability with $n=1 / 2$. The experiments were made with water drops on three different substrates with different equilibrium contact angles $\theta_{S}$ : clean glass (almost perfectly wetting, $\theta_{S} \sim 0^{\circ}$ ), coated glass $\left(\theta_{S} \sim 65^{\circ}\right.$ ), and Teflon-coated glass $\left(\theta_{S} \sim 115^{\circ}\right)$.

The aim of this work is to discuss the effect of the main parameters controlling the drop spreading: viscosity, density, surface tension, drop volume and surface wettability. The time evolution $r(t)$ of the base radius is analyzed as well as the time $t_{S}$ for the complete spreading of the drop. Each parameter has been varied in a large range while keeping constant the other parameters.

\section{Numerical procedure}

\subsection{Navier Stokes solver}

The numerical simulations reported in this work are performed with the volume of fluid (VoF) solver developed in the JADIM code
$[4,12]$. The one-fluid system of equations is obtained by introducing the one-fluid function $C$ used to localize one of the two phases. In this study, we define $C$ as $C=1$ in the drop (phase 1 ), and $C=0$ in the surrounding air (phase 2 ). The one-fluid function $C$ makes possible the definition of the one fluid variables $U=C U_{1}+(1-C) U_{2}$ for the velocity, $P=C P_{1}+(1-C) P_{2}$ for the pressure, $\rho=C \rho_{1}+(1-C) \rho_{2}$ for the density and $\mu=C \mu_{1}+(1-C) \mu_{2}$ for the viscosity. The position of the interface is then given by the transport equation:

$\frac{\partial C}{\partial t}+U . \nabla C=0$

The two fluids are assumed to be Newtonian and incompressible with no phase change. Under isothermal condition and in the absence of any surfactant, the surface tension is constant and uniform at the interface between the two fluids. In such case, the velocity field $U$ and the pressure $P$ satisfy the classical one-fluid formulation of the Navier-Stokes equations:

$\nabla \cdot U=0$

$\rho\left(\frac{\partial U}{\partial t}+U . \nabla U\right)=-\nabla P+\nabla . \Sigma+\rho g+F_{\sigma}$

where $\Sigma$ is the viscous stress tensor, $g$ is the gravity. $F_{\sigma}$ is the capillary contribution:

$F_{\sigma}=\sigma \nabla . n n \delta_{I}$

where $\sigma$ is the surface tension, $n$ denotes by arbitrary choice the unit normal of the interface going out from the drop and $\delta_{I}$ is the Dirac distribution associated to the interface.

The system of Eqs. (3)-(6) is discretized using the finite volume method. Time advancement is achieved through a third-order Runge-Kutta method for the viscous stresses. Incompressibility is satisfied at the end of each time step though a projection method. The overall algorithm is second-order accurate in both time and space. The volume fraction $C$ and the pressure $P$ are volumecentered and the velocity components are face-centered. Due to the discretization of $C$, it results a numerical thickness of the interface, the cells cut by the interface corresponding to $0<C<1$. The specific aspect of our approach compared to the classical VoF or Level Set methods [19-21] concerns the technique used to control the stiffness of the interface. In our approach no interface reconstruction or redistancing techniques are introduced. The interface location and stiffness are both controlled by an accurate transport algorithm based on FCT (Flux-Corrected-Transport) schemes [25]. This method leads to an interface thickness of about three grid cells due to the implementation of a specific procedure for the velocity used to transport $C$, in flow region of strong strain and shear [4]. The numerical description of the surface tension is one of the crucial points when considering systems where capillary effects control the interface shape. This interfacial force is solved using the classical CSF (continuum surface force) model developed by [5]. A classical problem of this formulation is the generation of spurious currents $[13,18]$. In order to decrease spurious currents intensity, a classical method introduced by [5] was employed, which consists in calculating the surface curvature from a smoothed density gradient, while the discretization of the delta function uses an un-smoothed density. The spurious currents in our code JADIM have been characterized by [12]. Their maximum magnitude evolves as $0.004 \sigma / \mu$, in agreement with other codes using the Brackbill's formulation.

\subsection{Numerical modeling of the contact angle}

The modeling used in this work aims to respect the physics at the contact line. It is based on two main considerations at the macroscopic scale: 


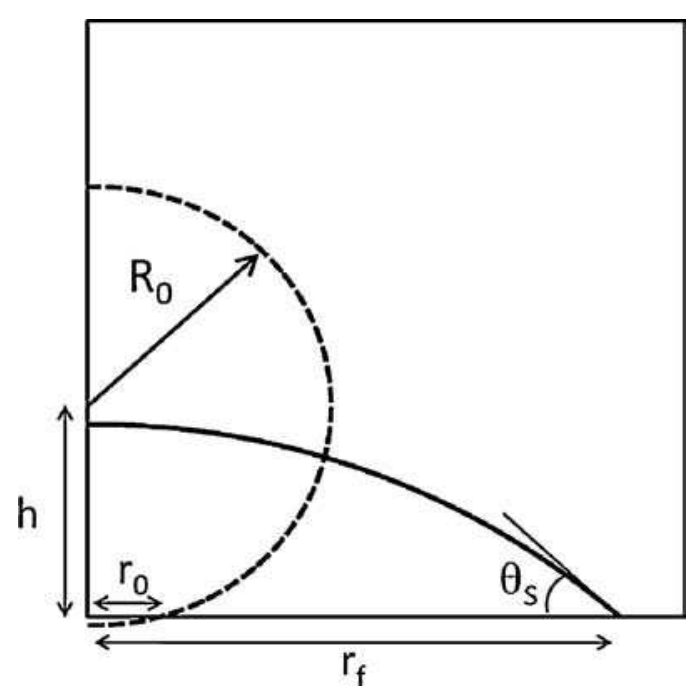

Fig. 1. Statement of the problem. Initial and final drop shape characterized by the base radius $r_{0}$ and $r_{f}$, respectively. The radial and vertical size are $L_{r}=L_{z}=3 R_{0} . h$ is the initial distance between the drop center and the wall.

- The wall condition seen by the fluid is a non slip condition.

- There is a direct relation between the interface velocity and the shape of the interface characterized by the apparent contact angle.

The objective is to solve the physic at the apparent scale $L$ where the contact angle is the apparent or dynamic contact angle. At this scale the fluid obeys a non slip condition for the resolution of the momentum equation:

$U_{W}=0$

Hydrodynamics at a smaller scale than $L$ has an important role since it links the apparent angle to the static angle. A sub-grid modeling of such effect needs to be introduced in the simulation. This is done by using the relation derived by Cox [8] in order to link the apparent angle to the static angle:

$g\left(\theta_{W}\right)=g\left(\theta_{S}\right)+\operatorname{CaLn}\left(\frac{L}{\lambda}\right)$

where $C a=\mu U_{c l} / \sigma$ is the contact line capillary number. The first Volume of Fluid node being located at $\Delta / 2$ from the wall, it follows that $L=\Delta / 2$. Considering the value of the observed slip length [14], we have used a fixed value $\lambda=10^{-9} \mathrm{~m}$ for the slip length in (4) in the simulations reported in this work. Note that experimental observations give also a recommendation for the choice of the macroscopic length $L$ consistent with the dynamic model used in our numerical modeling. Indeed one should have $\Delta / 2=L \approx 10^{-4}-10^{-5} \mathrm{~m}$.

The comparison between our modeling with other variant modelings, i.e. static contact angle versus dynamic contact angle, no slip condition versus slip condition, is presented in [16]. A grid and time convergence has been performed and discussed for the different possible models. These comparisons clearly stress the strong effect of the numerical modeling used for the simulation of dynamic contact lines as illustrated in the next section, where the simulations are compared with experiments for water and more viscous liquids.

\subsection{Comparison with experiments for water and viscous drops}

We consider a spherical droplet of volume $V=4 \pi R_{0}^{3} / 3$ deposited on an horizontal wall that enters in contact with an horizontal wettable surface at time $t=0$. All the numerical experiments reported in this work are described by Fig. 1. The computational domain is a square domain of equal radial and vertical extension

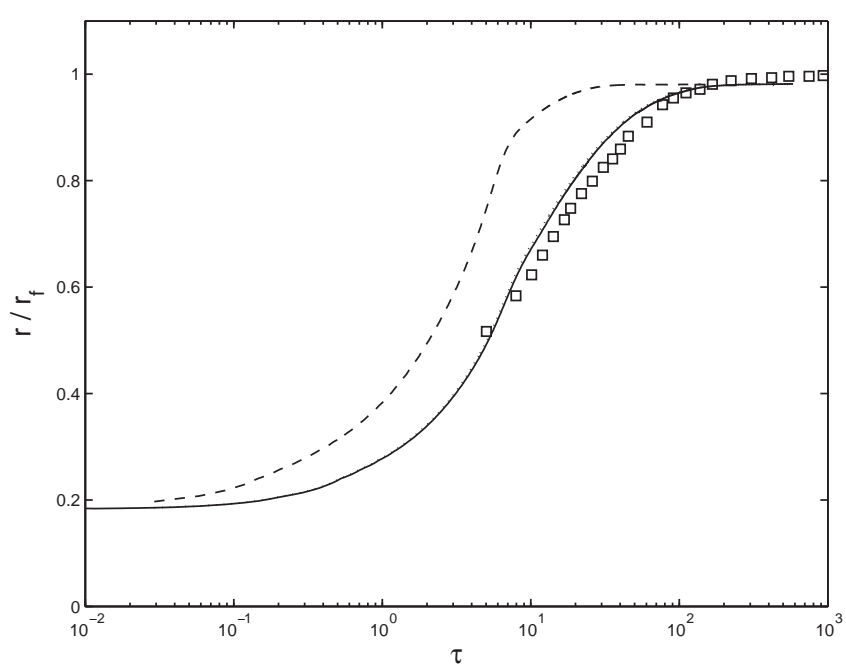

Fig. 2. Comparison with the experiments for viscous drop [15]. The normalized radius $r / r_{f}$ is reported versus the normalized time $\tau=\sigma t / \mu V^{1 / 3}$. Contact angle models used for the simulations: - Dynamic model, - - - static model

$L_{r}=L_{z}=3 R_{0}$. The axis of symmetry corresponds to the west boundary, the wetting conditions given by eq. (7-4) are imposed on the south boundary and classical wall conditions (no slip) are imposed on the two other boundaries (east and north). The initial condition corresponds to a drop initially located at the distance $h \leq R_{0}$ from the wall. The effect of this initial condition as well as the grid and time convergences have been performed [16] by considering different initial locations of the drop center $(h=0.95,0.98$, $0.99,0.995,0.998 R_{0}$ ), different grids (corresponding to $32,64,128$ and 167 nodes per radius) and different time steps ( $\Delta t=10^{-5}$ to $\left.\Delta t=10^{-8} \mathrm{~s}\right)$.

Squalane and water drops are considered for comparison with the experiments of Lavi \& Marmur [15] Winkels et al. and [24], respectively.

We first consider the experiments of [15]. They have considered the spreading of squalane drops with $R_{0}=1 \mathrm{~mm}$. The fluid properties are $\rho=809 \mathrm{~kg} / \mathrm{m}^{3}, \mu=0.034$ Pa s and $\sigma=0.032 \mathrm{~N} / \mathrm{m}$. The comparison between our simulations and the experiments is shown in Fig. 2. The normalized radius $r / r_{f}$ is reported versus the normalized time $\tau=\sigma t / \mu V^{1 / 3}$. The dynamic model is compared with the static model (a constant contact angle $\theta_{S}$ being imposed during all the simulation). A significant difference is observed between the dynamic model (4) and the static model $\left(\theta_{W}=\theta_{S}\right)$. The dynamic models give a satisfactory agreement with the experiments of [15].

We now consider the spreading of water drops and we compare our simulations with the experiments performed by [24] for millimeter-sized water droplets in air $\left(R_{0}=0.5 \mathrm{~mm}\right)$. We have selected the cases corresponding to very different contact angles $\theta_{S}=115^{\circ}$ and $\theta_{S} \approx 0^{\circ}$.

Based on preliminary tests [16], the comparison with the experiments performed by [24] are performed with a grid corresponding to 128 nodes per radius $R_{0}$ and a time step $\Delta t=10^{-7} \mathrm{~s}$. The initial position is $h=0.998 R_{0}$. The comparison is shown in Fig. 3 where the normalized contact line radial position $r / R_{0}$ is shown as function of the time normalized by the capillary-inertial time $t_{\rho}=\left(\rho R_{0}^{3} / \sigma\right)^{1 / 2}$. The agreement is very satisfactory for the two cases considered. In particular, the power law $t^{1 / 2}$ is clearly reproduced by the simulations. Consequently, our simulations do not show a noticeable effect of the surface wettability on the power law evolution in agreement with [24] but in opposition with [2]. Below, we consider in detail this point with additional simulations performed for a fluid ten times more viscous than water. Note also that our simulation reveals the oscillation of the contact line at the end of the 


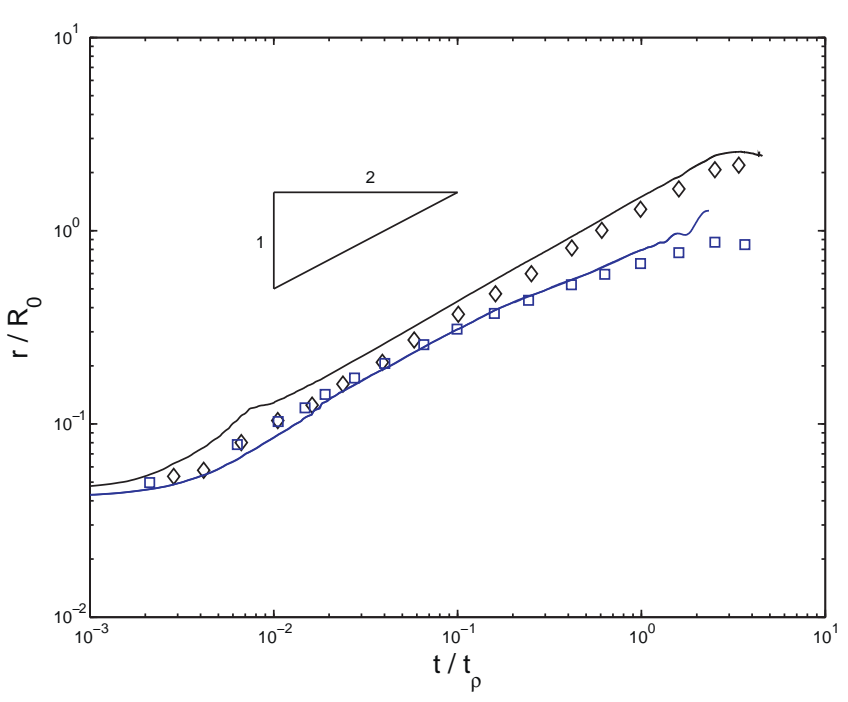

Fig. 3. Evolution of the contact line radial position $r / R_{0}$ as function of the normalized time $t /\left(\rho R_{0}^{3} / \sigma\right)^{1 / 2}$. Experiments [24]: ( $\left.\square\right) \theta_{S}=115^{\circ}$, ( $\left.\diamond\right) \theta_{S} \approx 0^{\circ}$. Numerical simulations: $---\theta_{S}=115^{\circ},-\theta_{S}=5^{\circ}$.

spreading. Such oscillation are not reported in the experiments of [24] and will be discussed in the following.

\section{Results}

We consider the effect of the main parameters controlling drop spreading: fluid properties (liquid density, liquid viscosity and surface tension), the drop size and the wetting properties of the surface. Each parameter is varied independently over a wide range of variation. All the simulations have been performed considering air as the surrounding fluid. As a consequence the density and viscosity ratio values (i.e. $\rho_{2} / \rho_{1}$ and $\mu_{2} / \mu_{1}$ ) are not constant but they always remain much smaller than unity so that there is no expected effect of both the viscosity and the density of the air on the spreading.

\subsection{Inertial-capillary regime of spreading}

We first consider low-viscous spreading. The density, the surface tension and the drop radius are varied independently while the viscosity is set fixed to the viscosity of water $\mu=0.001$ Pas. Fig. 4 shows the evolution of the contact line radius when varying the density from $\rho=100 \mathrm{~kg} / \mathrm{m}^{3}$ to $\rho=4000 \mathrm{~kg} / \mathrm{m}^{3}$ with the other parameters kept constant: $\sigma=0.072 \mathrm{~N} / \mathrm{m}, \mu=0.001$ Pas, $R_{0}=0.5 \mathrm{~mm}$ and $\theta_{S}=65^{\circ}$. When the density is increased the spreading time grows (the curves are translated to the right) since the inertia of the liquid to be accelerated by the moving contact line is more important. We notice that the same power law evolution $t^{1 / 2}$ is observed for all the range of density considered. The $t^{1 / 2}$ power law results from a capillary-inertial balance. Indeed, when the spherical drop of radius $R_{0}$ contacts the solid, the balance between the capillary pressure $\sim \sigma R_{0} / r^{2}$ and the inertial pressure $\sim \rho(d r / d t)^{2}$ gives after integration the following evolution:

$r(t) \sim\left(\frac{\sigma R_{0}}{\rho}\right)^{1 / 4} t^{1 / 2}$

Fig. 4 (bottom) confirms that the drop spreading is controlled by a balance between capillary and inertia, since the use of the time normalized using the capillary-inertia characteristic time $t_{\rho}=$ $\left(\rho R_{0}^{3} / \sigma\right)^{1 / 2}$ makes possible a good collapse of the different curves.

Fig. 4 also reveals contact line oscillations at both the early and the final stage of spreading. Indeed as shown in Fig. 5, the interface is significantly deformed by the propagation of capillary waves.
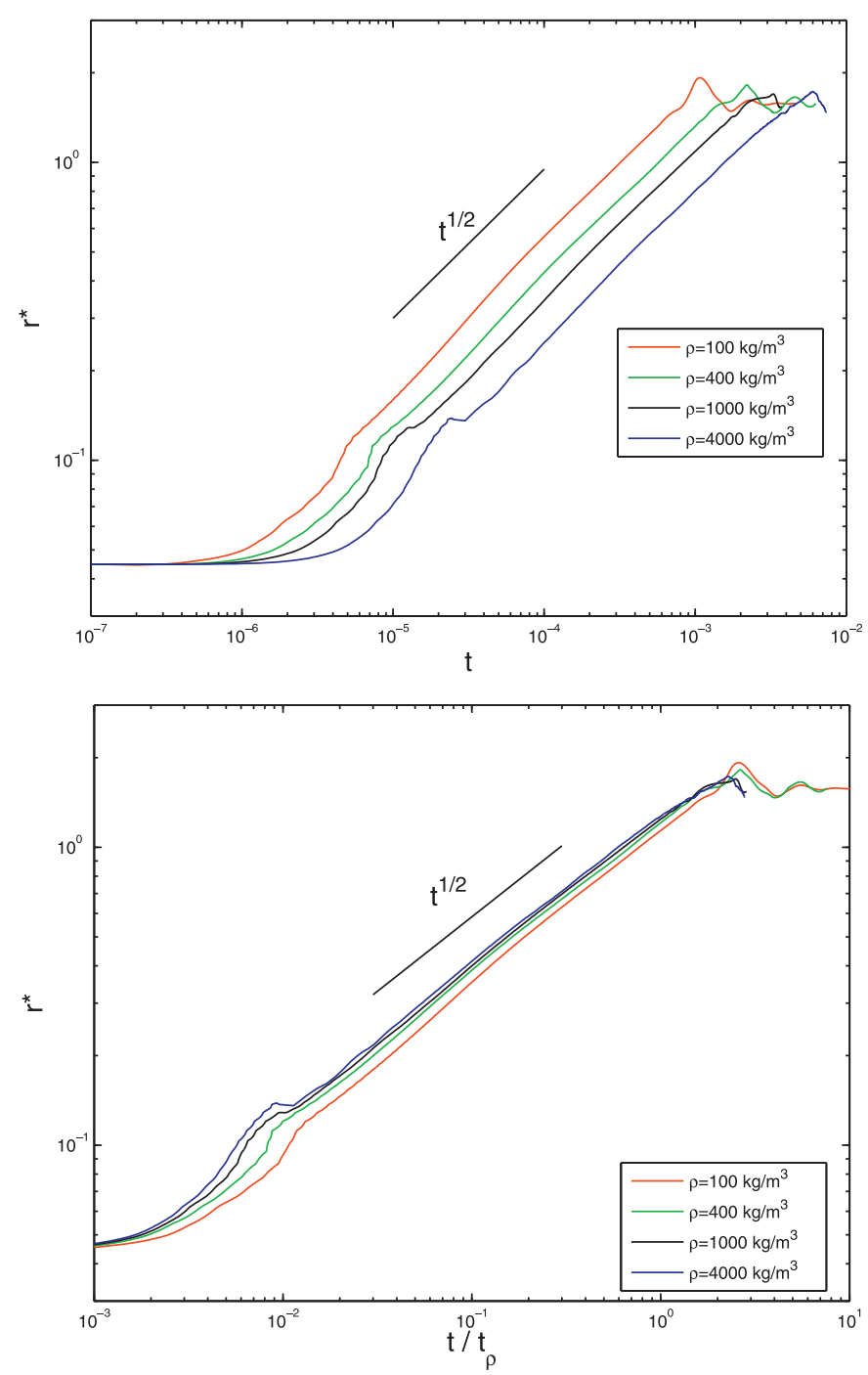

Fig. 4. Effect of the liquid density on the drop spreading for $\sigma=0.072 \mathrm{~N} / \mathrm{m}$, $\mu=0.001 \mathrm{Pas}, \theta_{S}=65^{\circ}$ and $R_{0}=0.5 \mathrm{~mm}$. The normalized contact line radius $r^{*}=\left(r-r_{0}\right) /\left(r_{f}-r_{0}\right)$ is reported as a function of (top) the dimensional time $t$ and (bottom) the normalized time $t /\left(\rho R_{0}^{3} / \sigma\right)^{1 / 2}$.

The appearance of a capillary wave that travels from the contact line to the top of the droplet has also been reported in the simulations reported by [11] using a diffuse interface method. This figure reports the evolution of the shape of a water drop for $\theta_{S}=65^{\circ}$. The sequence is presented line by line for the times given in the legend. The time steps are non regular in order to show particular events observed during the spreading. At the beginning of the spreading, only the contact line is moving. The top of the drop starts to move due to the capillary wave induced by the initial impulse generated at the contact line. Surprisingly this capillary wave induces the elevation of the top of the drop. As a consequence, combined with the displacement of the contact line, the drop is elongated into two orthogonal directions resulting in the generation of a pinch event (see 2 nd image ( $t=2.52 \mathrm{~ms}$ ), line 3 ). In this case, the pinch-off does not occurs resulting in the propagation of a relatively large amplitude wave. This capillary wave displaces again the top of the drop in the direction opposite to the wall. This generates a second pinch that results in the ejection of a small satellite droplet (see 2 nd image $(t=3.34 \mathrm{~ms})$, line 5$)$. Then, the satellite droplet impacts and coalesces with the drop generating again wave oscillations, explaining the final oscillations observed for the contact line in Fig. 4. Such regime of droplet ejection following the deposition of drops onto 

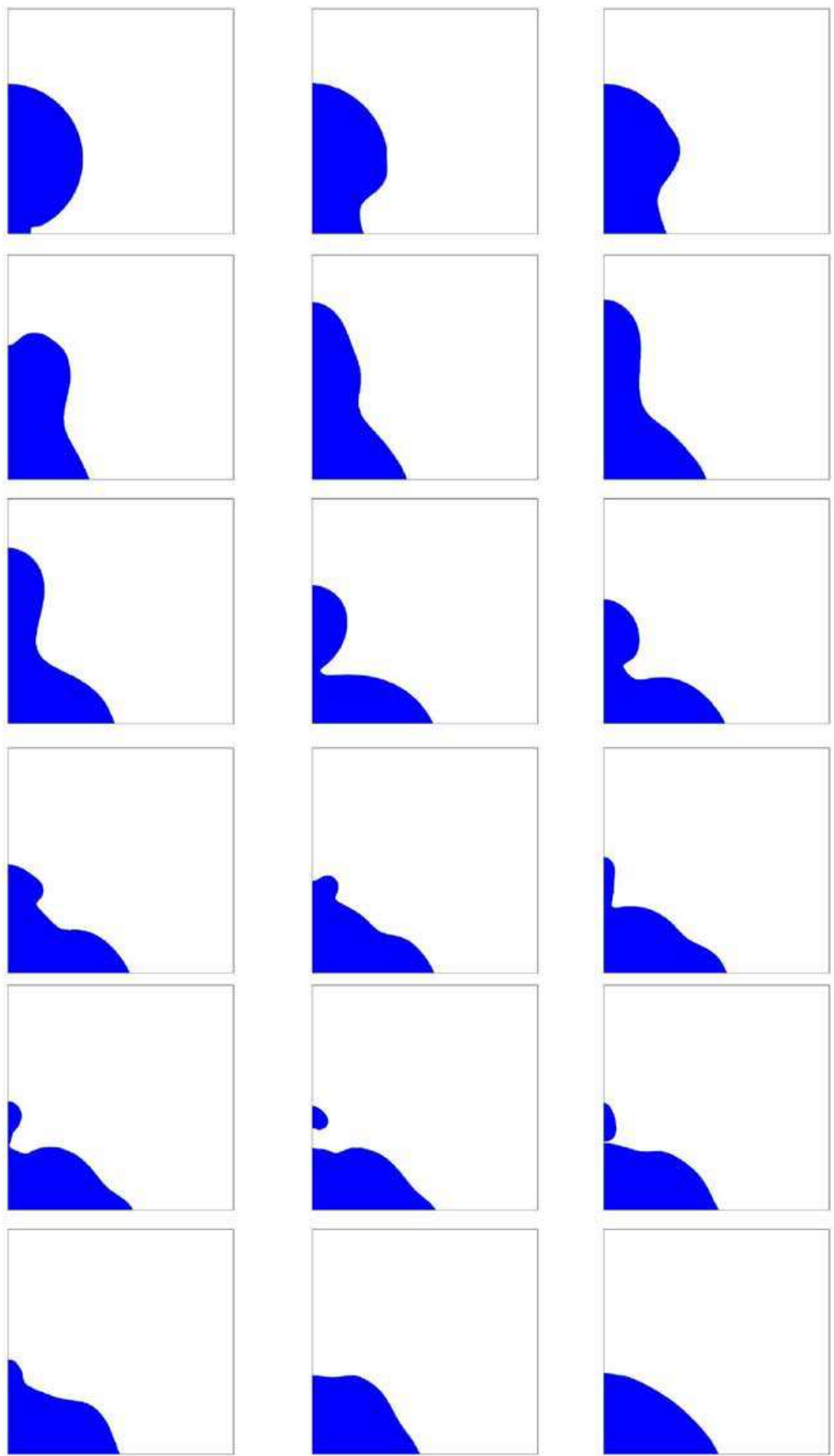

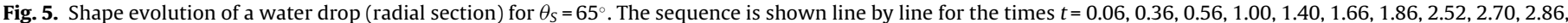
$2.98,3.12,3.28,3.34,3.52,3.60,4.80$ and $5.80 \mathrm{~ms}$.

a solid substrate has been identified in experiments and direct numerical simulations by [10] for smaller contact angles $\left(\theta_{S} \leq 65^{\circ}\right)$. We show here that the droplet ejection can also be observed for larger contact angles.

We illustrate in Fig. 6 the effect of the density on the droplet ejection. The simulations are shown for three densities $\rho=700 \mathrm{~kg} / \mathrm{m}^{3}$, $\rho=1000 \mathrm{~kg} / \mathrm{m}^{3}$ and $\rho=4000 \mathrm{~kg} / \mathrm{m}^{3}$. The images corresponding to the two successive pinch-offs and to the maximum height of the satellite droplet are shown. For $\rho=700 \mathrm{~kg} / \mathrm{m}^{3}$ two pinch events are observed but no droplet ejection is generated by the surface oscillation, while for $\rho=4000 \mathrm{~kg} / \mathrm{m}^{3}$ the droplet is ejected at the first pinch event.

The effect of the surface tension is now reported in Fig. 7. The surface tension is varied from $\sigma=0.007 \mathrm{~N} / \mathrm{m}$ to $\sigma=0.2 \mathrm{~N} / \mathrm{m}$. The other parameters are fixed to $\rho=10^{3} \mathrm{~kg} / \mathrm{m}^{3}, \mu=0.001 \mathrm{Pas}$, $R_{0}=0.5 \mathrm{~mm}$ and $\theta_{S}=65^{\circ}$. The spreading time is decreased when increasing the surface tension, since the initial impulse transmitted to the drop is increased. We also observe that the power law 


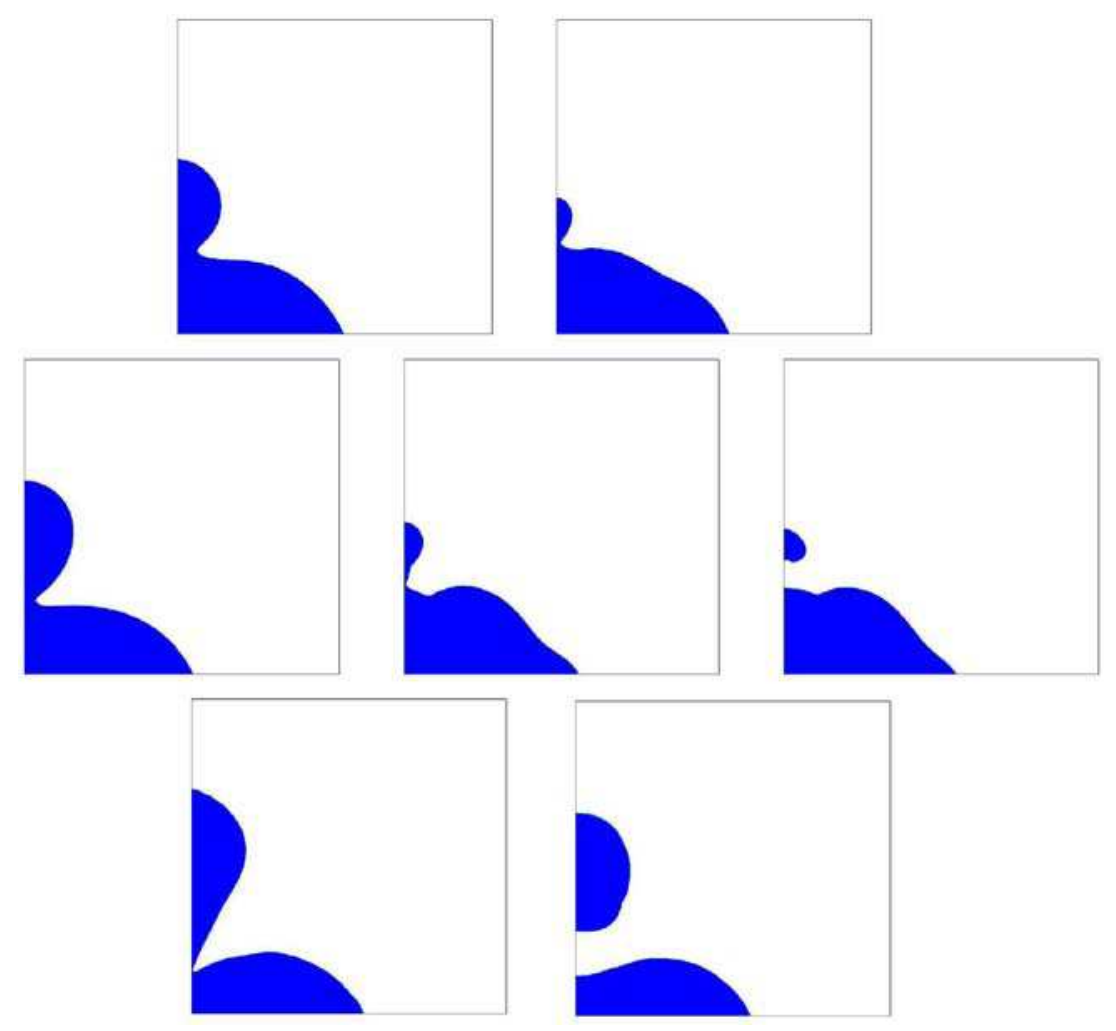

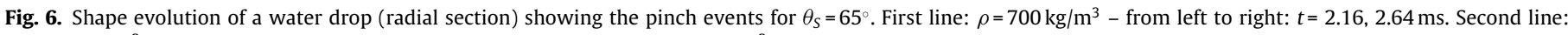
$\rho=1000 \mathrm{~kg} / \mathrm{m}^{3}-$ from left to right: $t=2.52,3.28,3.34 \mathrm{~ms}$. Third line: $\rho=4000 \mathrm{~kg} / \mathrm{m}^{3}-$ from left to right: $t=4.56,4.94 \mathrm{~ms}$.

evolution $t^{1 / 2}$ is not significantly affected by the change of the surface tension. Again the use of the normalized time $t /\left(\rho R_{0}^{3} / \sigma\right)^{1 / 2}$ makes possible the collapse of the curves and clearly reveals the capillary oscillations that impact the contact line spreading.

We report in Fig. 8 the spreading time $t_{S}$ for the contact line evolution shown in Figs. 4 and 7. The spreading times deduced from simulations for water drops with radii $R_{0}=0.5 \mathrm{~mm}$ and $R_{0}=5 \mathrm{~mm}$ are also reported. $t_{S}$ is shown as a function of the characteristic capillary-inertial time $t_{\rho}=\left(\rho R_{0}^{3} / \sigma\right)^{1 / 2}$. $t_{S}$ is defined as the time necessary to reach $98 \%$ of the final equilibrium radius $r_{f}$ of the contact line. The plot confirms that spreading is clearly controlled by the balance between inertia and capillarity. The spreading time can be estimated as:

$t_{S} \approx 1.7\left(\frac{\rho R_{0}^{3}}{\sigma}\right)^{1 / 2}$

The spreading time is thus increased when reducing the surface tension and increasing the drop density and the drop size.

\subsection{Viscosity effect}

We report in Fig. 9 the effect of the viscosity on the drop spreading. The viscosity is varied from $\mu=0.001 \mathrm{~Pa}$ s to $\mu=0.07 \mathrm{~Pa}$ s for $\rho=10^{3} \mathrm{~kg} / \mathrm{m}^{3}, \sigma=0.072 \mathrm{~N} / \mathrm{m}, R_{0}=0.5 \mathrm{~mm}$ and $\theta_{S}=65^{\circ}$. The spreading time is enlarged when increasing the viscosity (the curves being translated to the right) and the oscillations observed at the end of the spreading due to the propagation of capillary wave are damped. Despite the increase of the spreading time, the slope of the evolution at the early stage of the spreading increases from $t^{1 / 2}$ (water) to $t^{2 / 3}$ for the largest viscosity considered. But the duration of the power law regime is reduced because the end of the spreading is characterized by an exponential evolution of the form given by (2). For comparison, the Tanner law $t^{1 / 10}$ is also shown in the figure.
There is no clear evidence that this type of evolution is observed in our simulations during the final stage of the spreading. In fact, the static contact angle $\theta_{S}=65^{\circ}$ is too large to observe such a regime. For liquids more viscous than water, we expect that the evolution is controlled by a viscous-capillary balance. The viscous stress near the contact line can be estimated as $\sim \mu(d r / d t) / \ell$ where $\ell$ is the characteristic length controlling the shear stress at the contact line. Assuming an evolution of the form $\ell=R_{0}^{1-p} r^{p}$, the balance between the viscous stress and the capillary pressure $\sim \sigma R_{0} / r^{2}$ gives, after integration, the observed $t^{2 / 3}$ evolution:

$r(t) \sim\left(\frac{\sigma R_{0}^{1 / 2}}{\mu}\right)^{2 / 3} t^{2 / 3}$

for $p=3 / 2$ corresponding to $\ell \sim R_{0}^{-1 / 2} r^{3 / 2}$. The corresponding viscous-capillary characteristic time $t_{\mu}=\mu R_{0} / \sigma$ is used in Fig. 4 (bottom) to normalize the time. For a viscosity larger than $0.01 \mathrm{~Pa}$, a satisfactory collapse of the curves is observed indicating the transition to a spreading regime controlled by the balance between capillarity and viscosity.

The evolution of the spreading time $t_{S}$ is reported in Fig. 10 as a function of the viscous-capillary characteristic time $t_{\mu}=\mu R_{0} / \sigma$. Two different behaviors are clearly observed. For low viscosity, $t_{S}$ is found to evolve as $t_{\mu}^{1 / 5}$ (i.e. $\mu^{1 / 5}$ since all the other parameters are not varying). We have no explanation of the particular evolution $t_{S} \propto \mu^{1 / 5}$ revealed by the simulation for low-viscous drops. For larger viscosities an expected linear evolution $t_{S} \propto t_{\mu}$ is observed. The linear behavior results from the linearity of the governing equations (Stokes equation) at low Reynolds number. The viscous-capillary spreading time can be estimated using the relation

$t_{S} \approx 55 \frac{\mu R_{0}}{\sigma}$ 

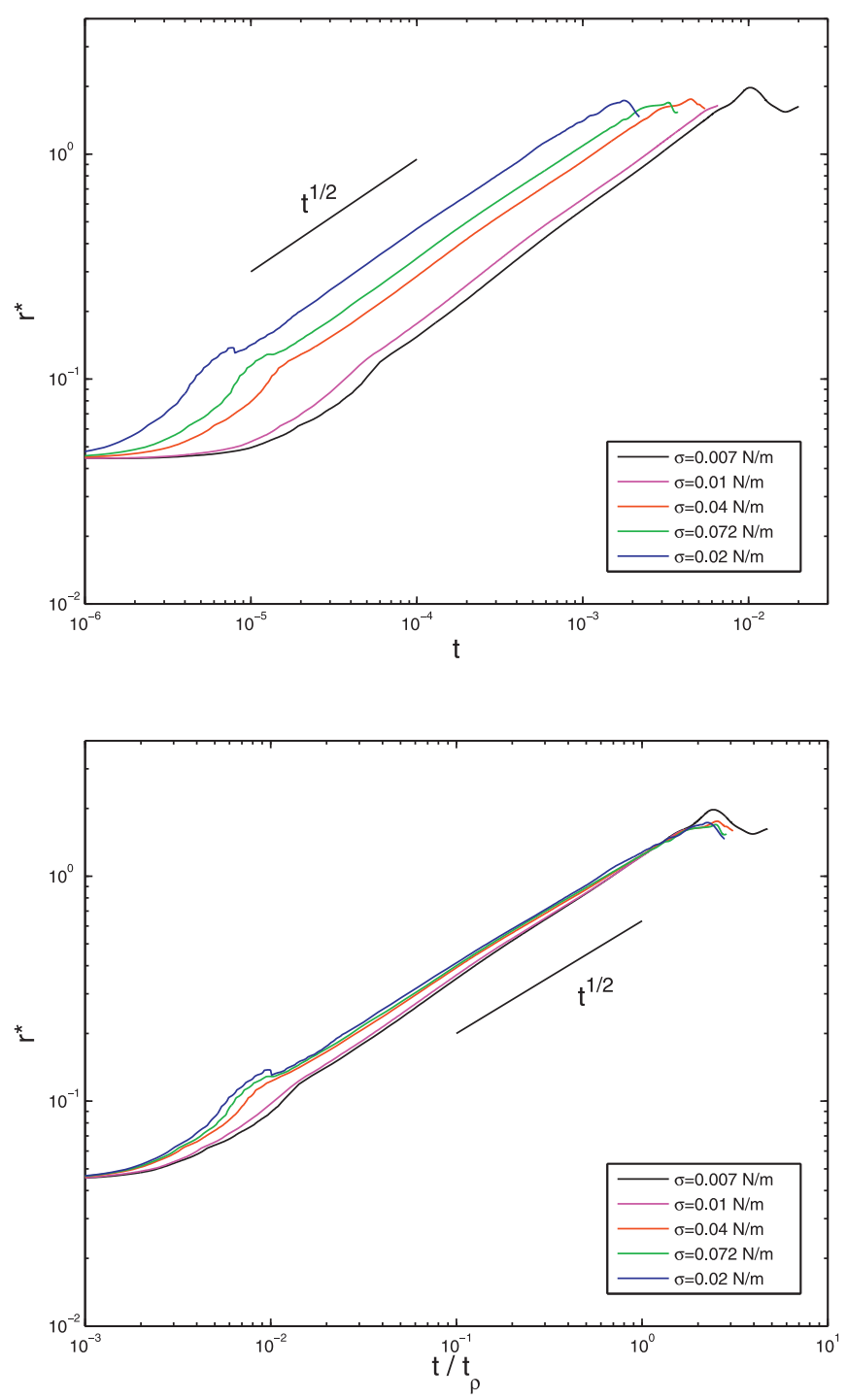

Fig. 7. Effect of the surface tension on the drop spreading for $\rho=10^{3} \mathrm{~kg} / \mathrm{m}^{3}$, $\mu=0.001 \mathrm{Pas}, R_{0}=0.5 \mathrm{~mm}$ and $\theta_{S}=65^{\circ}$. The normalized contact line radius $r^{*}=\left(r-r_{0}\right) /\left(r_{f}-r_{0}\right)$ is reported as a function of (top) the dimensional time $t$ and (bottom) the normalized time $t /\left(\rho R_{0}^{3} / \sigma\right)^{1 / 2}$.

\subsection{Contact angle effect}

We finally discuss the effect of the static contact angle $\theta_{S}$ on drop spreading. Figs. 3, 4 and 7 show that the $t^{1 / 2}$ power law is not affected by the surface wettability at the beginning of the spreading, when considering a fluid having the same viscosity as water for a large range of contact angles $\left(\theta_{S}=5^{\circ}, 65^{\circ}\right.$ and $\left.115^{\circ}\right)$, in agreement with the experiments reported by [24] for water drops. The wetting conditions considered by $[24]\left(\theta_{S}=5^{\circ}, 65^{\circ}\right.$ and $\left.115^{\circ}\right)$ are now simulated for a fluid with a viscosity ten times larger than that of water ( $\mu=0.01 \mathrm{~Pa}$ ), the other parameters being kept the same as for water $\left(\sigma=0.072 \mathrm{~N} / \mathrm{m}, \rho=10^{3} \mathrm{~kg} / \mathrm{m}^{3}\right)$ and $R_{0}=0.5 \mathrm{~mm}$. Additional wetting conditions $\left(\theta_{S}=140^{\circ}\right.$ and $\left.\theta_{S}=160^{\circ}\right)$ have also been considered.

Fig. 11 clearly reveals that the wetting condition has now a significant effect on the spreading rate. When the contact angle is increased the slope seems to decrease in agreement with the experiments of [2]. However, if the radius $r$ is normalized using the radius reached at the end of the inertial-capillary regime, all the curves collapse on a single curve showing a $t^{1 / 2}$ evolution independent on the contact angle $\theta_{S}$ in agreement with the experiments of [24].

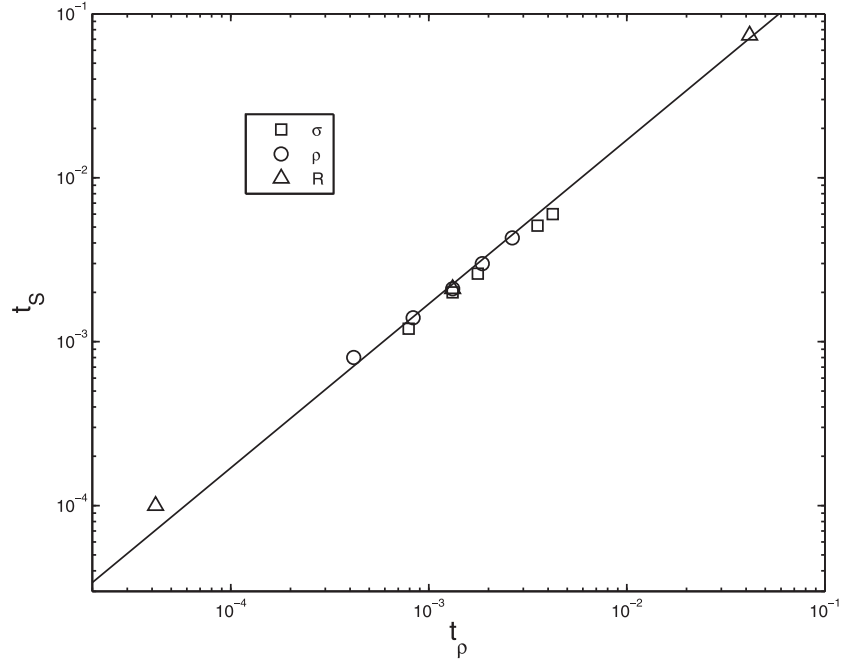

Fig. 8. Spreading time $t_{S}$ versus the capillary-inertial time $t_{\rho}=\left(\rho R_{0}^{3} / \sigma\right)^{1 / 2}$ when varying the liquid density, the surface tension and the drop radius. (०) $\rho$ is varying from $100 \mathrm{~kg} / \mathrm{m}^{3}$ to $4000 \mathrm{~kg} / \mathrm{m}^{3}$ for $\mu=0.001 \mathrm{~Pa} \mathrm{~s}, \sigma=0.072 \mathrm{~N} / \mathrm{m}, R_{0}=0.5 \mathrm{~mm}$ and $\theta_{S}=65^{\circ}$. ( $\left.\square\right) \sigma$ is varying from $0.007 \mathrm{~N} / \mathrm{m}$ to $0.2 \mathrm{~N} / \mathrm{m}$ for $\rho=10^{3} \mathrm{~kg} / \mathrm{m}^{3}, \mu=0.001$ Pa s, $R_{0}=0.5 \mathrm{~mm}$ and $\theta_{S}=65^{\circ}$. ( $\left.\triangle\right) R_{0}$ is varying from $0.5 \mathrm{~mm}$ to $5 \mathrm{~mm}$ for $\rho=10^{3} \mathrm{~kg} / \mathrm{m}^{3}$, $\mu=0.001 \mathrm{~Pa} \mathrm{~s}, \sigma=0.072 \mathrm{~N} / \mathrm{m}$ and $\theta_{S}=65^{\circ}$. The continuous line represents the relation $t_{S}=1.7 t_{\rho}$
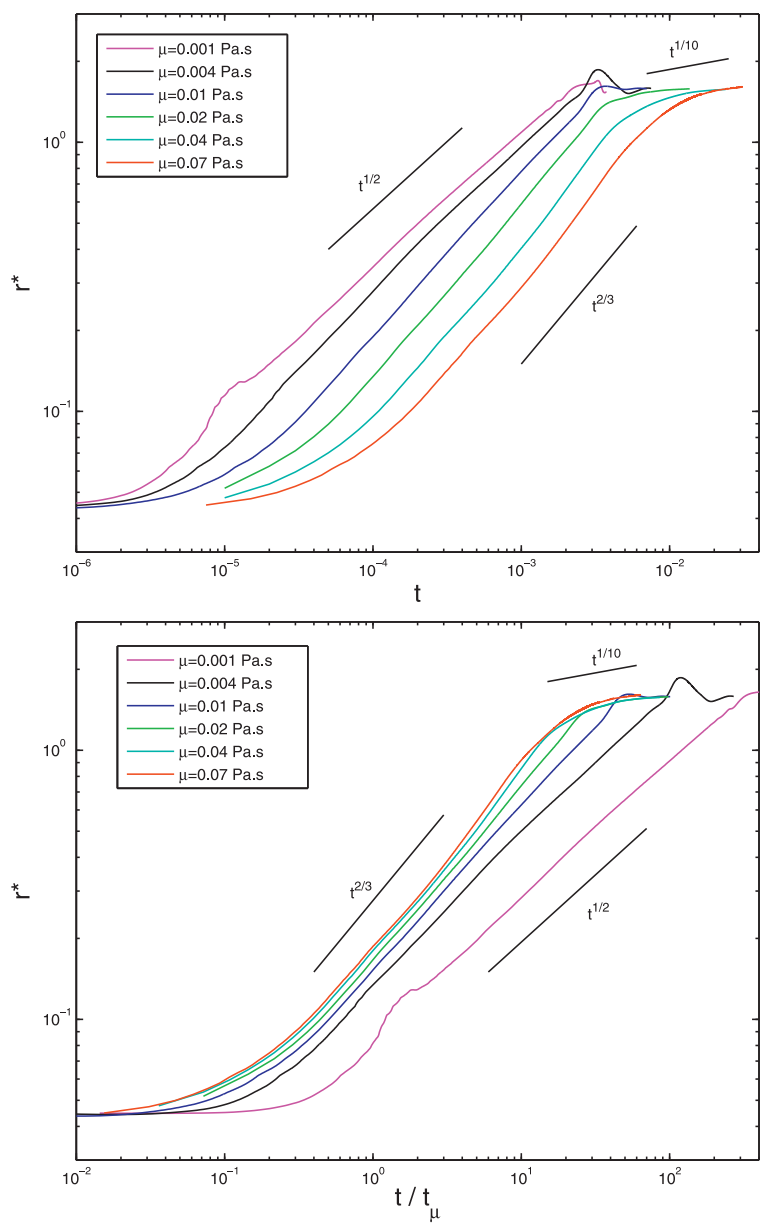

Fig. 9. Effect of the liquid viscosity on the drop spreading for $\sigma=0.072 \mathrm{~N} / \mathrm{m}$, $\rho=10^{3} \mathrm{~kg} / \mathrm{m}^{3}, R_{0}=0.5 \mathrm{~mm}$ and $\theta_{S}=65^{\circ}$. The normalized radius $r^{*}=\left(r-r_{0}\right) /\left(r_{f}-r_{0}\right)$ is shown versus (top) the dimensional time $t$ and (bottom) the normalized time $t / t_{\mu}$. 


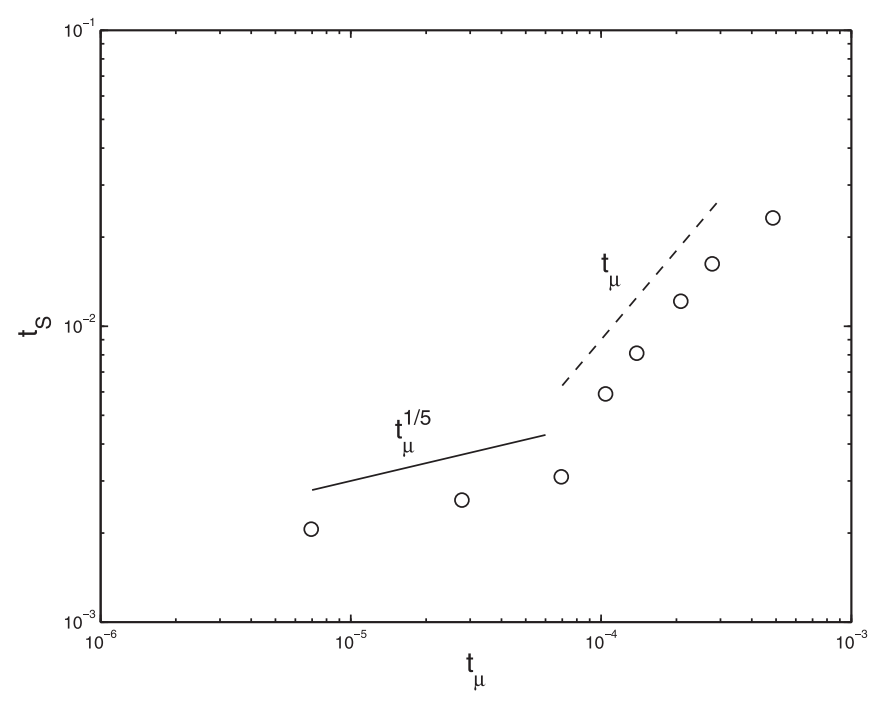

Fig. 10. Spreading time $t_{S}$ versus the viscous-capillary characteristic time $t_{\mu}=\mu R_{0} / \sigma$ for $\sigma=0.072 \mathrm{~N} / \mathrm{m}, \rho=10^{3} \mathrm{~kg} / \mathrm{m}^{3}, R_{0}=0.5 \mathrm{~mm}$ and $\theta_{S}=65^{\circ} .(-) t_{S} \propto t_{\mu}^{1 / 5},(---)$ $t_{S} \propto t_{\mu}$.

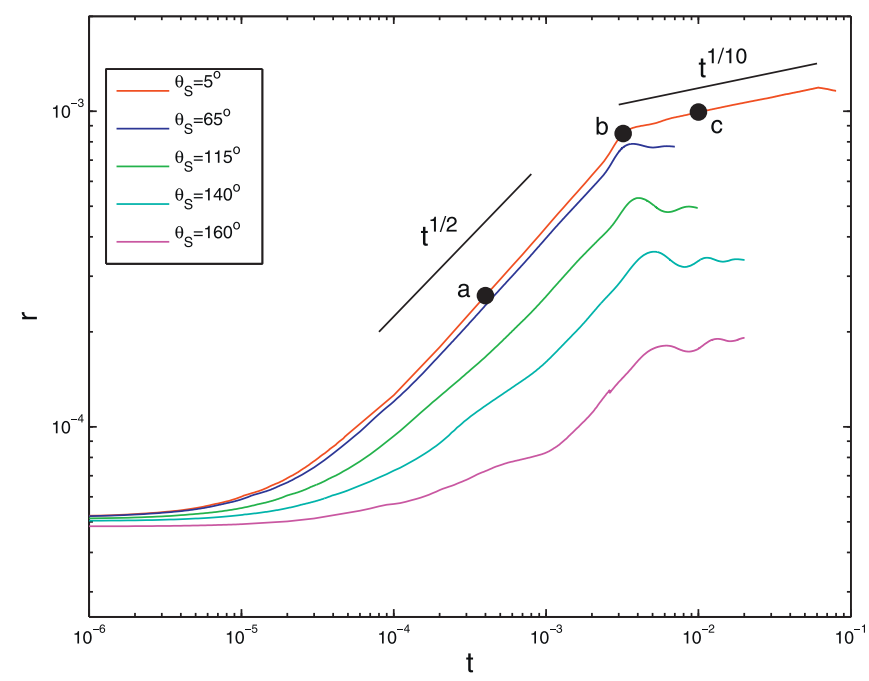

Fig. 11. Effect of the contact angles $\theta_{S}$ on the time evolution of the drop base radius $r$ for $\mu=0.01$ Pa s, $\sigma=0.072 \mathrm{~N} / \mathrm{m}, \rho=10^{3} \mathrm{~kg} / \mathrm{m}^{3}$ and $R_{0}=0.5 \mathrm{~mm}$. The drop radial sections corresponding to $\mathrm{a}, \mathrm{b}$ and $\mathrm{c}$ are shown in Fig. 12.

For the smallest contact angle $\theta_{S}=5^{\circ}$, we observe after the $t^{1 / 2}$ evolution a clear $t^{1 / 10}$ evolution characteristic of the Tanner Law. A similar behavior has been reported in the simulations of [11] where a regime close to the Tanner's law is observed when increasing the viscosity while a regime close to $t^{1 / 2}$ is observed at the early stage of the spreading. Fig. 12 shows the shape of the drop during the $t^{1 / 2}$ evolution, during the $t^{1 / 10}$ evolution and at the transition between these two regimes. The corresponding times are depicted by bullets in Fig. 11. A spherical cap is clearly identified during the $t^{1 / 10}$ evolution.

In the limit of a very thin spherical cap, the contact angle $\theta_{W}$, the drop volume $V$ and the contact line radius $r$ are linked by the geometrical relation $\theta_{W} \approx 4 V / \pi r^{3}$. In the limit of a small dynamic contact angle with $\theta_{S} \approx 0$, the Cox relation (4) can be expressed as:

$\theta_{W}^{3}=9 \frac{\mu}{\sigma} \frac{d r}{d t} \ln (L / \lambda)$

Combining these two relations and integrating in time, the radius of the contact line is found to follow the famous Tanner law:

$r(t) \sim R_{0}\left(\frac{t}{t_{\mu}}\right)^{1 / 10}$

For spreading drops, such evolution is thus observed for small contact angles once the drop has reached a spherical cap shape as shown in Fig. 12c. In the limit of $\theta_{S} \rightarrow 0$, the base radius of the spherical cap can be expressed as $r_{f} \approx R_{0} \theta_{S}^{-1 / 3}$ and it follows an estimation of the spreading time:

$t_{S} \approx t_{\mu} \theta_{S}^{-10 / 3}$

For the simulations reported in Fig. 11, relation (14) gives $t_{S} \approx 0.2 \mathrm{~s}$ which is a correct order of magnitude for $\theta_{S}=5^{\circ}$. The corresponding viscous-capillary spreading time given by relation (12) is two orders of magnitude smaller $\approx 0.0038 \mathrm{~s}$, but in agreement with the evolution reported for the larger contact angles $\left(\theta_{S}=65^{\circ}, 115^{\circ}\right.$, $140^{\circ}$ and $160^{\circ}$ ).

\section{Conclusion}

We have considered the spreading of a drop on a horizontal wettable surface by numerical simulation. In our numerical modeling of moving contact lines, the apparent contact angle is given by the [8] relation while a zero slip length is imposed for the momentum calculation. The simulations have been satisfactorily compared with the experiments of [15] for squalane and with the experiments of [24] for water. The effects of the main parameters controlling the drop spreading have been studied separately. For viscosity of the order of that of water, the $t^{1 / 2}$ power law has been recovered at the early stage of the spreading for all the contact angles considered in agreement with the experiments of [24]. When increasing the viscosity, the early stage of the spreading is still characterized by a power law evolution but with an increasing exponent in agreement with the experiments of [2]. Both the viscosity and the contact angle are then shown to impact the line expansion. In particular, considering the spreading of a viscous drop on a highly wettable substrate $\left(\theta_{S}=5^{\circ}\right)$, we observe two successive power law evolutions. The capillary-inertial regime $r \propto t^{1 / 2}$ is followed by the
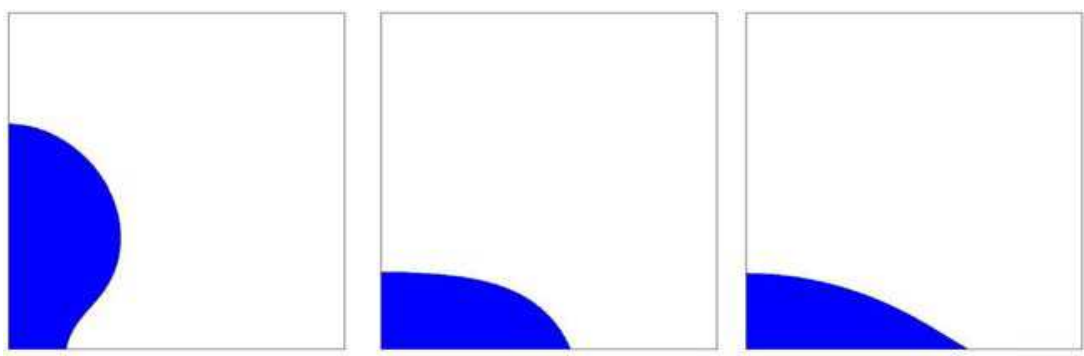

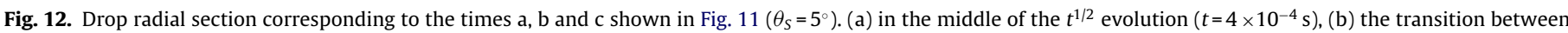
the $t^{1 / 2}$ and the $t^{1 / 10}$ evolution $\left(t=3.2 \times 10^{-3} \mathrm{~s}\right)$ and $(\mathrm{c})$ in the middle of the Tanner law evolution $\left(t=10^{-2}\right)$. 
Tanner $r \propto t^{1 / 10}$ evolution once the droplet has reached a spherical cap shape. Our simulations show that the combined effect of the viscosity and the contact angle plays an important role in the spreading of a drop. They make consistent the different experimental observations (a priori contradictory) concerning the effect of the wetting conditions. enddocument

\section{References}

[1] A.-L. Biance, C. Clanet, D. Quéré, First steps in the spreading of a liquid droplet, Phys. Rev. E 69 (016301) (2004).

[2] J. Bird, S. Mandre, H. Stone, Short-time dynamics of partial wetting, Phys. Rev. Lett. 100 (234501) (2008).

[3] D. Bonn, J. Eggers, J. Indekeu, J. Meunier, E. Rolley, Wettinga and spreading, Rev. Mod. Phys. 81 (739) (2009).

[4] T. Bonometti, J. Magnaudet, An interface capturing method for incompressible two-phase flows. validation and application to bubble dynamics, Int. J. Multiphase Flow 33 (2007) 109-133.

[5] J. Brackbill, D. Kothe, C. Zemach, A continuum method for modeling surface tension, J. Comput. Phys. 100 (1992) 335-354.

[6] A. Cazabat, M. Cohen Stuartt, Dynamics of wetting: effects of surface roughness, J. Phys. Chem. 90 (1986) 5845-5849.

[7] L. Chen, E. Bonaccurso, M.E.R. Shanahan, Inertial to viscoelastic transition in early drop spreading on soft surfaces, Langmuir 29 (6) (2013) 1893-1898.

[8] R. Cox, The dynamics of the spreading of liquids on a solid surfaces. Part 1 : viscous flow, J. Fluid Mech. 168 (169) (1986).

[9] P.-G. de Gennes, Wetting: statics and dynamics, Rev. Mod. Phys. 57 (827)(1985).

[10] H. Ding, E. Li, F. Zhang, Y. Sui, P. Spelt, S. Thoroddsen, Propagation of capillary waves and ejection of small droplets in rapid droplet spreading, J. Fluid Dyn. 697 (2012) 92-114.
[11] H. Ding P.D.M. Spelt, Inertial effects in droplets spreading: a comparison between diffuse-interface and level-set simulations, J. Fluid Mech. 576 (2007) 287-296.

[12] J. Dupont, D. Legendre, Numerical simulations of static and sliding drop with contact angle hysteresis, J. Comput. Phys. 229 (2010) 2453-2478.

[13] B. Lafaurie, C. Nardone, R. Scardovelli, S. Zaleski, G. Zanetti, Modelling merging and fragmentation in multiphase flows with surfer, J. Comput. Phys. 113 (1994) 134-147.

[14] E. Lauga, M. Brenner, H. Stone, Handbook of Experimental Fluid Dynam-ics Ch. 19, in: Microfluidics: The No-Slip Boundary Condition, Springer, New-York, 2007, pp. 1219-1240.

[15] B. Lavi, A. Marmur, The exponential power law: partial wetting kinetics and dynamic contact angles, Colloids Surf. 250 (2004).

[16] M. Maglio, Numerical simulation of spreading, sliding and coalescing drops on surfaces, Institut National Polytechnique de Toulouse, 2012, Ph.D. thesis.

[17] A. Marmur, Equilibrium and spreading of liquids on solid surfaces, Adv. Colloid Interface Sci. 19 (75) (1983).

[18] S. Popinet, S. Zaleski, A front-tracking algorithm for accurate representation of surface tension, Int. J. Numer. Methods Fluids 30 (1999) 775-793.

[19] R. Scardovelli, S. Zaleski, Direct numerical simulation of free surface and interfacial flow, Ann. Rev. Fluid Mech. 31 (567) (1999).

[20] J. Sethian, Level set methods and fast marching methods, Cambridge University Press, Cambridge, 1999

[21] M. Sussman, E. Fatemi, P. Smereka, S. Osher, A level set approach fro computing solutions in incompressible two-phase flows, J. Comput. Fluids 27 (1998) 663-680.

[22] L. Tanner, The spreading of silicone oil drops on horizontal surfaces, J. Phys. D: Appl. Phys. 12 (1979) 1473-1484

[23] O. Voinov, Hydrodynamics of wetting, J. Fluid Dyn. 11 (1976).

[24] K. Winkels, J. Weijs, A. Eddi, J. Snoeijer, Initial spreading of low-viscosity drops on partially wetting surfaces, Phys. Rev. E 85 (055301(R)) (2012).

[25] S. Zalesak, Fully multidimensional flux-corrected transport algorithms for fluids, J. Comput. Phys. 31 (1979) 335-362. 